\title{
French pharma firms terminate trials of bumetanide for autism
}

\section{BY PETER HESS}

1 OCTOBER 2021

Two phase 3 clinical trials of the diuretic drug bumetanide were terminated by their sponsors on 7 September after interim data showed the drug performs no better than a placebo at easing autism traits.

Despite the lackluster results, the researchers investigating bumetanide say it is too soon to give up.

"The inclusion criteria should have been a bit tougher," says co-lead investigator Yehezkel BenAri, president and co-founder of the French biotech firm Neurochlore, which holds the patent for bumetanide as an autism treatment.

Neurochlore launched both trials in 2018 in partnership with French pharmaceutical company Servier Laboratories. The two companies joined forces in 2017 after Neurochlore reported preliminary findings that bumetanide eased autism traits in two different groups of children. Animal studies suggest the drug corrects an imbalance of excitatory and inhibitory signals in the brain. This imbalance is hypothesized to contribute to autism traits.

After the new negative finding, however, Servier ended the partnership, leaving Neurochlore without funding to continue the work.

One of the trials had enrolled 211 autistic children aged 2 to 6 years, and the other included 211 autistic children and teenagers aged 7 to 17 . At the start of both trials, participants were randomly assigned to take either 0.5 milligrams of bumetanide or a placebo twice per day for 6 months; during a 6-month 'open-label' extension study, all participants in both groups were supposed to knowingly take the drug.

But by the end of the placebo-controlled portion of each study, the participants who took 


\section{Spectrum | Autism Research News}

https://www.spectrumnews.org

bumetanide fared no differently than controls on a clinician questionnaire called the Childhood Autism Rating Scale, the study's primary outcome measure. They also fared no better on a parent questionnaire called the Social Responsiveness Scale, one of the study's secondary outcome measures.

\section{Missed target:}

There is a great temptation to blame methodological problems for a trial's failure, but the simplest explanation would be that bumetanide is not helpful in autism, says Jeremy VeenstraVanderWeele, professor of psychiatry at Columbia University, who was not involved in the trials.

Regardless, Ben-Ari and his colleagues are reanalyzing their data to see whether any subgroups of participants showed meaningful differences in autism traits after treatment.

A 2020 trial of bumetanide for autism led by an independent team also missed its primary endpoint, assessed via the Social Responsiveness Scale. But participants showed improvements in repetitive behaviors, the study's secondary outcome, and a post-hoc analysis suggested that a subset of autistic children and teenagers had improved on both measures. Previous Neurochlore trials in 2012 and 2017 showed that bumetanide leads to a significant reduction in autism traits, but not necessarily for children with severe traits.

Anecdotal feedback from the parents of participants in the halted trials supports this idea, too, BenAri says.

Limiting future trials of bumetanide to participants who are most likely to respond could help researchers detect more significant differences between treatment and placebo groups, says Hilgo Bruining, associate professor of psychiatry at Amsterdam University Medical Centre in the Netherlands, who led the 2020 study.

To that end, Bruining's team has developed a method that uses electroencephalography to identify people who stand to benefit from the drug, based on its proposed mechanism of action. In a randomized, double-blind, placebo-controlled trial that included 82 participants with autism, several brain-wave markers - including the ratio between excitatory and inhibitory signaling predicted with 92 percent accuracy which participants would show a greater reduction in repetitive behaviors in response to bumetanide. The researchers are conducting a post-trial study to further hone the prediction algorithm in those participants and others.

Ben-Ari is seeking research partners at academic institutions and says Neurochlore needs new funding sources to conduct further trials on bumetanide. "At the moment, the main problem is finance."

Cite this article: https://doi.org/10.53053/UJIK1183 